\title{
The Cygno experiment for Dark Matter direct detection
}

\author{
S. Piacentini ${ }^{a, b, *}$ for the CYGNO collaboration ${ }^{\dagger}$ \\ ${ }^{a}$ Dipartimento di Fisica Sapienza Università di Roma, Roma, I-00185, Italy \\ ${ }^{b}$ Istituto Nazionale di Fisica Nucleare, Sezione di Roma, Roma, I-00185, Italy \\ E-mail: stefano.piacentini@uniroma1.it
}

Innovative experimental techniques are needed to further search for dark matter Weakly Interacting Massive Particles (WIMPs). The ultimate limit is represented by the ability to efficiently reconstruct and identify nuclear and electron recoil events at the experimental energy threshold. Gaseous Time Projection Chambers (TPC) with optical readout are very promising candidates thanks to the 3D event reconstruction capability of the TPC technique and the high sensitivity and granularity of last generation light sensors. The Cygno experiment is pursuing this technique by developing a TPC operated with $\mathrm{He}: \mathrm{CF}_{4}$ gas mixture at atmospheric pressure equipped with a Gas Electron Multipliers (GEM) amplification stage that produces visible light collected by a scientific CMOS camera. Events are then reconstructed with an innovative multi-stage pattern recognition algorithm based on advanced clustering techniques. In this contribution, we present the performances of several prototype detectors assessed by exposing them to radioactive sources. We show that good energy and spatial resolution as well as discriminating power between nuclear and electron recoils is achieved in the keV energy range. Finally, we discuss the plan to build a $1 \mathrm{~m}^{3}$ demonstrator expected to be installed and operated at LNGS in 2023/24. This experimental campaign aims at proving the scalability of such a detector concept to a bigger apparatus able to significantly extend our knowledge about DM and neutrinos.

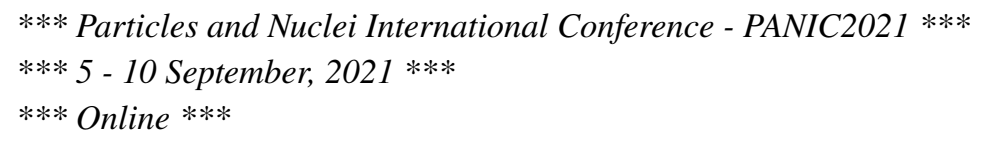

\footnotetext{
${ }^{*}$ Speaker

${ }^{\dagger}$ F. D. Amaro, E. Baracchini, L. Benussi, S. Bianco, C. Capoccia, M. Caponero, D.S. Cardoso, G. Cavoto, A. Cortez, I.A. Costa, E. Dané, G. Dho, F. Di Giambattista, E. Di Marco, G. D’Imperio, F. Iacoangeli, H.P. Lima Júnior, A.S. Lopes Júnior, G. Maccarrone, R.D.P. Mano, M. Marafini, R.R. Marcelo Gregorio, D. Marques, G. Mazzitelli, A.G. McLean, A. Messina, C.M.B. Monteiro, R.A. Nobrega, I.F. Pains, E. Paoletti, L. Passamonti, S. Pelosi, F. Petrucci, S. Piacentini, D. Piccolo, D. Pierluigi, D. Pinci, A. Prajapati, F. Renga, R.J.C. Roque, F. Rosatelli, A. Russo, J.M.F. dos Santos, G. Saviano, N. Spooner, R. Tesauro, S. Tomassini, S. Torelli
} 


\section{Introduction}

The CYGNO project aims to construct a large detector for high precision 3D tracking of rare, low energy, nuclear recoils (NR) induced by Dark Matter (DM). An ideal experimental technique for this task is the Time Projection Chamber (TPC). Weakly interactive massive DM particles can interact with ordinary matter nuclei, inducing NR. Assuming the Standard galactic Halo Model, the energy of the induced NR is expected to be of the order of few keVs, sufficient to produce a detectable track inside a TPC. In order to properly detect these events, a set of experimental challenges has to be overcome, due to the expected low DM signal rate and the small energy transferred to the nuclei. The Cygno collaboration is developing a strategy based on the use of high sensitivity scientific CMOS (sCMOS) cameras to capture the NR events occurring inside a TPC filled with a He: $\mathrm{CF}_{4}$ gas mixture kept at $1 \mathrm{~atm}$ pressure [1]. A stack of 3 Gas Electron Multipliers (GEMs) is used to amplify the ionization produced during an event and as consequence visible light is emitted by the $\mathrm{CF}_{4}$ scintillator. The scintillation photons are detected by the camera and by a set of fast photosensors, namely PhotoMultiplier Tubes (PMTs). The high granularity and very low noise camera acquires images on the plane perpendicular to the electron drift direction, while the PMTs allow 3D track reconstruction. In addition, due to the optical coupling, the sensors are outside the TPC, making it possible the readout of large surfaces with relatively small photosensors.

Cygno initial R\&D phase started in 2015 and was devoted to the assessment of the gas mixture and to find the best readout strategy. Several prototypes were built, from ORANGE [2] (with an active volume of $0.1 \mathrm{~L}$ ) to the latest LIME prototype [1] $(50 \mathrm{~L})$. Cygno is now in the so called PHASE-0: LIME prototype, currently operating above ground at the Laboratori Nazionali di Frascati (LNF), will be moved this year to underground at the Laboratori Nazionali del Gran Sasso (LNGS), paving the way for the so called PHASE-1, aiming the construction of a $1 \mathrm{~m}^{3}$ detector, as upgrade of the LIME prototype. This detector will be commissioned at LNGS in 2023/2024. The final aim of the collaboration is to build a larger detector of $30-100 \mathrm{~m}^{3}$.

\section{LEMON performance}

The CYGNO experimental approach has been studied with the LEMON prototype [3]: a $7 \mathrm{~L}$ active volume TPC with a $20 \mathrm{~cm}$ drift region, laterally limited by an elliptical field cage, and on the sides by a cathode electrode and a stack of 3 GEM foils [4] $\left(20 \times 24 \mathrm{~cm}^{2}\right)$. The scintillation light is detected by a PMT on the cathode side and by the sCMOS camera, a Hamamatsu Orca-Flash 4.0 with a $1.33 \times 1.33 \mathrm{~cm}^{2}$ and $2048 \times 2048$ pixels sCMOS sensor, on the GEM side.

In order to evaluate the detector energy resolution and efficiency, the $5.9 \mathrm{keVee} \mathrm{X}$-ray line emitted by a ${ }^{55} \mathrm{Fe}$ source [3] was used. The sCMOS data shows an energy resolution of $12 \%$, average light yield of $514 \pm 63$ photons per $\mathrm{keV}$ and a sensor noise below $400 \mathrm{eV}$, allowing operating with a $1 \mathrm{keV}$ threshold, see Fig. 1. The PMT data show a similar behavior, with an energy resolution of $16 \%$. We measured $x-y$ position resolutions of $100 \mu \mathrm{m}$ and $300 \mu \mathrm{m}$ for events close to the GEMs and $20 \mathrm{~cm}$ away from them, respectively. This difference is due to the transverse diffusion during the drift, which can be used to extract the clusters $z$ position, with $15 \%$ resolution [1] The longitudinal diffusion during the drift influences the time width of the signal: this information has then been 

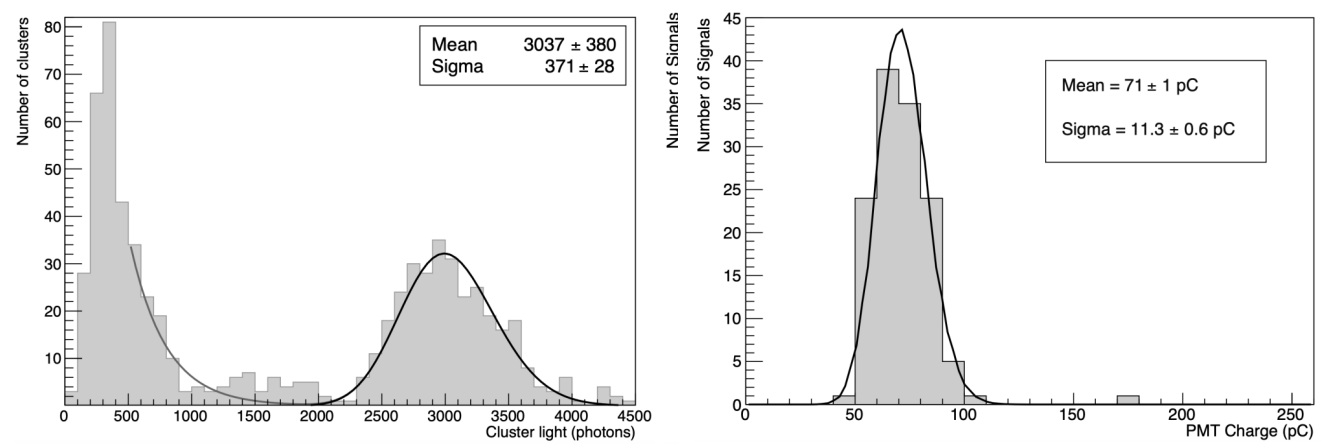

Figure 1: Left: distribution of the reconstructed clusters with the SCMOS by exposing the LEMON prototype to a ${ }^{55} \mathrm{Fe}$ source. Right: integrated charge of the signals collected by the PMT by exposing the LEMON prototype to a ${ }^{55} \mathrm{Fe}$ source.

used to extract the $z$ position using events detected by the PMT, providing approximately the same $z$ position resolution of $15 \%$.

An AmBe neutron source was used to assess LEMON response to NR events [5]. For the reconstruction, we developed a custom iterative DBSCAN + GAC (Geodesic Active Countur) pattern recognition algorithm [6], able to find and connect the ionization clusters from the images, and to provide track parameters such as track length and width, total collected light and light density $\delta$. Finally, an event recognition algorithm takes these parameters and separates NR induced by AmBe neutrons from electronic recoils (ER) induced by the 5.9 keVee X-rays. Just by cutting on $\delta$, for energy deposits around $5.9 \mathrm{keVee}$, the algorithm is able to discriminate ER from NR, with background rejection of $96 \%$ against ER and a signal efficiency of $40 \%$ for NR.

\section{LIME: the latest prototype towards the $1 \mathrm{~m}^{3}$ CYGNO demonstrator}

LIME, our latest prototype, is characterized by a $50 \mathrm{~cm}$ drift length and a $33 \times 33 \mathrm{~cm}^{2}$ sensitive area, for an active volume of $50 \mathrm{~L}$. The optical readout is done by a sCMOS camera improved version, the Hamamtsu Orca-Fusion, and by 4 PMTs, placed this time on the GEMs side. The new camera has a better CMOS sensor $(2304 \times 2304$ pixels $)$, higher quantum efficiency and lower noise. Thanks to its good properties, we are able to obtain an energy resolution of $15 \%$ at 5.9 keVee with LIME, similar to the one of LEMON but for a bigger volume. In addition, due to the reduced noise, we are able to operate with a lower threshold of $0.7 \mathrm{keV}$. The detector, designed, constructed and commissioned on surface at LNF, will be moved underground at LNGS by 2021, with an additional $10 \mathrm{~cm}$ copper and $0.5 \mathrm{~m}$ water shielding.

Once LIME is underground, the collaboration will enter PHASE-1, aimed at the construction of a $1 \mathrm{~m}^{3}$ CYGNO demonstrator. It will consist of 2 TPC's separated by a common cathode, each TPC closed by a $3 \times 3$ array of 3-GEMs stacks. Each of the 183 -GEM stacks will have the same optical readout of the LIME detector. For this purpose we are developing the trigger and data acquisition systems needed in view of this new larger detector, to be installed at LNGS by 2024 . 


\section{Conclusions}

The goal of the Cygno collaboration is to build a large GEM based TPC detector for directional dark matter direct detection. The strategy chosen by the collaboration is to use an optical readout made up of sCMOS cameras and fast photodetectors to measure the scintillation light produced inside a TPC filled with a He: $\mathrm{CF}_{4}$ gas mixture at atmospheric pressure. Due to several prototypes development, it has been possible to validate the technology and to assess its performance. In particular, the LEMON prototype showed very good energy and position resolutions in the keV energy region, confirmed in the larger LIME prototype thanks to a more advanced sCMOS camera sensor. In addition, the development of a custom clustering and reconstruction algorithm allows discriminating the ER background from the NR signal with good efficiency. The collaboration is finalizing the commissioning of the 50 L LIME prototype, currently above ground at LNF but that will soon be moved underground at LNGS by 2021. The next steps are the construction of the $1 \mathrm{~m}^{3}$ Cygno demonstrator that will be installed at LNGS by 2024 .

\section{Acknowledgments}

We want to thank General Services and Mechanical Workshops of Laboratori Nazionali di Frascati (LNF) and Laboratori Nazionali del Gran Sasso (LNGS) for their precious work and L. Leonzi (LNGS) for technical support. This project has received fundings under the European Union's Horizon 2020 research and innovation programme from the European Research Council (ERC) grant agreement No 818744. This project is supported by the Italian Ministry of Education, University and Research through the project PRIN: Progetti di Ricerca di Rilevante Interesse Nazionale "Zero Radioactivity in Future experiment" (Prot. 2017T54J9J).

\section{References}

[1] F.D. Amaro et al., The CYGNO Experiment, Instruments, 6, (Jan. 2022), [arXiv : 2202 . 05480]

[2] M. Marafini, V. Patera, D. Pinci, A. Sarti, A. Sciubba, and E. Spiriti, ORANGE: A high sensitivity particle tracker based on optically read out GEM, Nucl. Instrum. Meth. A, 865, (2017), 285-288

[3] E. Baracchini et al., Stability and detection of a GEM-based Optical Readout TPC with He/CF 4 gas mixtures, JINST, 15 (2020), no. 10 P10001, [arXiv: 2007. 00608]

[4] M. Marafini, V. Patera, D. Pinci, A. Sarti, A. Sciubba, and N. M. Torchia, Study of the Performance of an Optically Readout Triple-GEM, IEEE Transactions on Nuclear Science, 65 (Jan., 2018), 604-608.

[5] E. Baracchini et al, Identification of low energy nuclear recoils in a gas TPC with optical readout, Meas. Sci. and Tech., 32 (2020) 025902, [arXiv : 2007 . 12508]

[6] E. Baracchini et al, A density-based clustering algorithm for the CYGNO data analysis, JINST, 15 (2020), no. 12 T12003, [arXiv: 2007. 01763] 